\title{
Electrically and Mechanically Tunable Electron Spins in Silicon Carbide Color Centers
}

\author{
Abram L. Falk, Paul V. Klimov, Bob B. Buckley, Viktor Ivády, Igor Abrikosov, Greg \\ Calusine, William F. Koehl, Adam Gali and David D. Awschalom
}

\section{Linköping University Post Print}

\section{Tweet}

N.B.: When citing this work, cite the original article.

Original Publication:

Abram L. Falk, Paul V. Klimov, Bob B. Buckley, Viktor Ivády, Igor Abrikosov, Greg Calusine, William F. Koehl, Adam Gali and David D. Awschalom, Electrically and Mechanically Tunable Electron Spins in Silicon Carbide Color Centers, 2014, Physical Review Letters, (112), 18, 187601. http://dx.doi.org/10.1103/PhysRevLett.112.187601

Copyright: American Physical Society http://www.aps.org/

Postprint available at: Linköping University Electronic Press http://urn.kb.se/resolve?urn=urn:nbn:se:liu:diva-107840 


\title{
Electrically and Mechanically Tunable Electron Spins in Silicon Carbide Color Centers
}

\author{
Abram L. Falk, ${ }^{1,2}$ Paul V. Klimov, ${ }^{1,2}$ Bob B. Buckley, ${ }^{2}$ Viktor Ivády, ${ }^{3,4}$ Igor A. Abrikosov, ${ }^{4}$ Greg Calusine, ${ }^{2}$ \\ William F. Koehl, ${ }^{1,2}$ Ádám Gali, ${ }^{3,5}$ and David D. Awschalom ${ }^{1,2, *}$ \\ ${ }^{1}$ Institute for Molecular Engineering, University of Chicago, Chicago, Illinois 60637, USA \\ ${ }^{2}$ Center for Spintronics and Quantum Computation, University of California, Santa Barbara, Santa Barbara, California 93106, USA \\ ${ }^{3}$ Wigner Research Centre for Physics, Institute for Solid State Physics and Optics, Hungarian Academy of Sciences, \\ PO Box 49, H-1525 Budapest, Hungary \\ ${ }^{4}$ Department of Physics, Chemistry, and Biology, Linköping University, SE-581 83 Linköping, Sweden \\ ${ }^{5}$ Department of Atomic Physics, Budapest University of Technology and Economics, Budafoki út 8., H-1111 Budapest, Hungary
}

(Received 26 November 2013; published 5 May 2014)

\begin{abstract}
The electron spins of semiconductor defects can have complex interactions with their host, particularly in polar materials like $\mathrm{SiC}$ where electrical and mechanical variables are intertwined. By combining pulsed spin resonance with $a b$ initio simulations, we show that spin-spin interactions in $4 H$-SiC neutral divacancies give rise to spin states with a strong Stark effect, sub-10 ${ }^{-6}$ strain sensitivity, and highly spin-dependent photoluminescence with intensity contrasts of 15\%-36\%. These results establish SiC color centers as compelling systems for sensing nanoscale electric and strain fields.
\end{abstract}

DOI: 10.1103/PhysRevLett.112.187601

PACS numbers: 76.30.Mi, 42.50.Ex, 71.70.Ej

Silicon carbide is increasingly recognized as a potential material host for quantum-information and sensing technologies that leverage advanced semiconductor processing alongside solid-state spin control [1-9]. Much like nitrogenvacancy (NV) centers in diamond [10], neutral divacancies in $\mathrm{SiC}$ have ground-state electronic spins [1,2] that exhibit optical addressability [11-14] and long spincoherence times that persist up to elevated temperatures, even up to room temperature for certain defect forms [5,7]. The NV center in diamond is currently being developed for applications ranging from quantum communication [15] to nanoscale nuclear magnetic resonance $[16,17]$. The extensive uses of $\mathrm{SiC}$ in industry, including wafer-scale growth [18], high-power devices, and substrates for epitaxially grown GaN [19] and graphene [20], could propel such applications and many others [21-23] forward. Underlying the success of such advancements will be an improved understanding of how spins in semiconductor defects interact with their host crystal.

In this Letter, we demonstrate that the spin states of neutral divacanices and related defect states in $4 \mathrm{H}-\mathrm{SiC}$ are highly sensitive to electrical and mechanical perturbations of their host. Their electric field response is 2-7 times stronger than that $[24,25]$ of NV spins in diamond, and they exhibit significant spin-dependent photoluminescence (PL) contrast for high-fidelity spin readout. Moreover, ac strainsensing protocols demonstrated here lead to an optimized sensitivity projected to be in the $10^{-7} / \sqrt{\mathrm{Hz} \cdot \mathrm{N}}$ range, where $N$ is the number of interrogated spins. Although electric- and strain-field effects on $\mathrm{SiC}$ spins are interrelated due to SiC's piezoelectricity, our $a b$ initio simulations disentangle these two effects and attribute the strong spin response to electric fields to the high electron polarizability in $\mathrm{SiC}$. Because our techniques are based on point defects and measure intrinsic quantities, their applicability extends down to the nanometer scale. In the future, they could be applied towards sensing intracellular electric fields, integrating nanoscale sensing into $\mathrm{SiC}$ bioelectronics [26], or coupling spins to $\mathrm{SiC}$ nanomechanical resonators [27].

Neutral divacancies, consisting of neighboring $\mathrm{C}$ and $\mathrm{Si}$ vacancies, exist in four inequivalent forms in $4 H-\mathrm{SiC}$, distinguished by whether vacancies are located on the hexagonal $(h)$ or quasicubic $(k)$ lattice sites [5]. These forms have been alternatively labeled as PL1-PL4 [5,7], P6/P7 centers (in electron paramagnetic resonance studies) $[1,2,28]$, and the UD2 lines (in PL studies) [12]. Their electronic ground states are spin triplets and described by the Hamiltonian [24,29]:

$H=h D \sigma_{z}^{2}+g \mu_{B} \boldsymbol{\sigma} \cdot \mathbf{B}-E_{x}\left(\sigma_{x}^{2}-\sigma_{y}^{2}\right)+E_{y}\left(\sigma_{x} \sigma_{y}+\sigma_{y} \sigma_{x}\right)$,

where the defect axis is aligned along $\mathbf{z}, h$ is Planck's constant, $g=2.0$ is the electron $g$ factor, $\mu_{B}$ is the Bohr Magneton, $\boldsymbol{\sigma}$ is the vector of spin-1 Pauli matrices, $\mathbf{B}$ is the magnetic field, and $D, E_{x}$, and $E_{y}$ are the zero-magneticfield splitting parameters. These terms can be expanded as $D=D^{0}+d_{\|} F_{z}+e_{\|} \varepsilon_{z}$ and $E_{x, y}=E_{x, y}^{0}+d_{\perp} F_{x, y}+e_{\perp} \varepsilon_{x, y}$, where the $D^{0}$ and $E_{x, y}^{0}$ terms are the crystal-field splittings in the absence of applied strain and electric fields, $d_{\|}$and $d_{\perp}$ are the Stark-coupling parameters of the ground-state spin to an electric field (F) that is, respectively, parallel and perpendicular to the defect axis, $e_{\|}$and $e_{\perp}$ are the straincoupling parameters, and $\varepsilon$ is the effective strain field defined in Ref. [29]. PL1 and PL2 (the $h h$ and $k k$ divacancies, respectively) are oriented along the $\mathrm{SiC}$ 

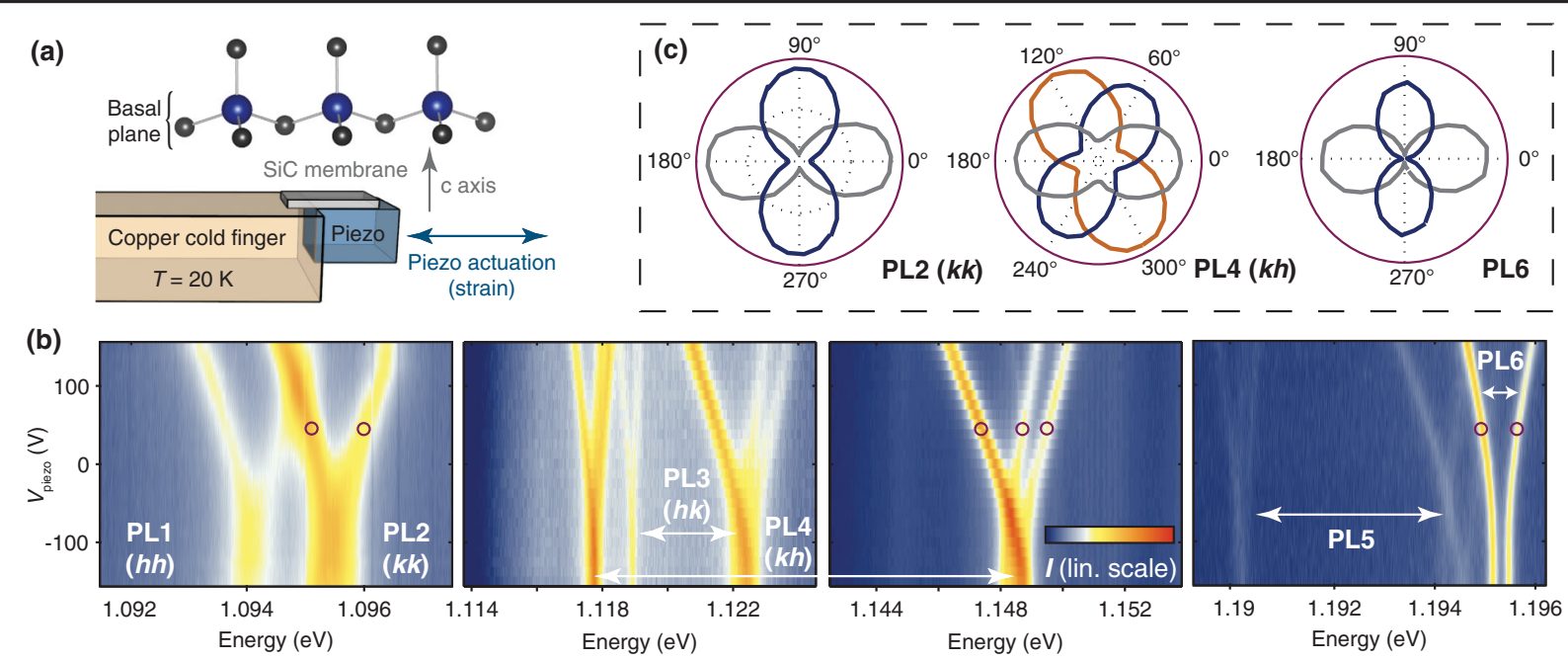

FIG. 1 (color). (a) A $4 \mathrm{H}$-SiC membrane is epoxied to the top of a piezo actuator, which applies strain to the SiC membrane as it stretches. (b) PL spectra as a function of $V_{\text {piezo }}$ (strain) at $T=20 \mathrm{~K}$, showing that the optical transitions of SiC defects can be tuned with strain. The applied strain splits the ZPL optical transitions, with the $c$ axis-oriented defects (PL1, PL2, and PL6) bifurcating and the basal-oriented defects (PL3, PL4, and PL5) trifurcating. (c) Polarization dependence of the PL from the strain-split ZPL branches, measured at the points indicated by purple circles in (b). The analyzed polarization is in the plane perpendicular to the $c$ axis.

$c$ axis and have $C_{3 v}$ symmetry (i.e., $E_{x, y}^{0}=0$ ). PL3 and PL4 (the $h k$ and $k h$ divacancies, respectively) are oriented along basal planes, at $109.5^{\circ}$ from the $c$ axis. This orientation reduces their symmetry to $C_{1 h}$, resulting in nonzero $E_{x, y}^{0}$ and thus broken degeneracy between all three spin sublevels at zero magnetic field.

Our experiments use high-purity semi-insulating $4 \mathrm{H}$-SiC wafers, purchased from Cree Inc., in which neutral divacancies are incorporated during crystal growth. We thinned $500 \mu \mathrm{m}$-thick chips of $\mathrm{SiC}$ down to $50 \mu \mathrm{m}$-thick membranes and used epoxy to mount them on top of piezo actuators, which are, in turn, mounted to copper cold fingers for cryogenic operation. The neutral divacancies' zero-phonon line (ZPL) optical transitions can be seen as peaks in their PL spectra [12] when we illuminate a SiC membrane with $1.27 \mathrm{eV}$ light at a temperature $T=20 \mathrm{~K}$. Each inequivalent divacancy has a distinct ZPL energy ranging from $1.0-1.2 \mathrm{eV}$. In addition, two other observed species (labeled PL5 and PL6) have similar optical and spin transition energies to the neutral divacancies [5], but the defects with which they are associated have not been identified.

Microwave radiation for electron spin resonance is supplied by waveguide antennae on the chip or below the sample [5,7]. The piezo actuation applies tensile strain to the $\mathrm{SiC}$ membrane transverse to the $c$ axis [Fig. 1(a)], an estimated $5 \times 10^{-7}$ strain $/ V_{\text {piezo }} \pm 40 \%$ (see the Supplemental Material [30]), where $V_{\text {piezo }}$ is the voltage applied to the piezo. Controls assure that electric fields within the measurement volume due to $V_{\text {piezo }}$ are too weak to interfere with any measurements.

As strain is applied, the energy of each defect species' ZPL splits and shifts as much as $2.3 \mathrm{meV}$, or $550 \mathrm{GHz}$
[Fig. 1(b)]. The ZPLs corresponding to the $c$ axis-oriented defects bifurcate, with the two resulting branches having orthogonally polarized PL [Fig. 1(c)]. This splitting, approximately $12 \mathrm{eV} /$ strain, reflects the reduction of the $C_{3 v}$ symmetry of the $c$ axis-oriented defects, whose doubly degenerate excited state orbitals at zero strain are predicted [2] to closely match the structure of $\mathrm{NV}$ centers in diamond [31,32]. In contrast, the basal-oriented defects have highly split ZPLs even at zero strain due to the crystal field. Each ZPL branch of the basal-plane-oriented defects trifurcates as strain is applied, with the polarization from each branch offset by $120^{\circ}$. This splitting indicates that, as expected, strain breaks the symmetry between orientations of defects in the basal plane that are equivalent at zero strain.

Applying transverse strain to the $\mathrm{SiC}$ membrane also shifts the defect's electronic spin transition energies. We measure these shifts with optically detected magnetic resonance (ODMR). Here, we read out the ground-state spin by exploiting the defects' spin-dependent $\left(m_{s}=0\right.$ vs $\left.m_{s}= \pm 1\right)$ PL intensity $(I)$ and monitoring the changes to $I(\Delta I)$ as an applied microwave field rotates spins via electron spin resonance [5,7]. The $\Delta I$ resonances as the frequency of the applied microwave field is swept correspond to spin transition energies [Fig. 2(a)].

As we apply uniaxial strain transverse to the $c$ axis, the average frequency of the two $\Delta m_{s}= \pm 1$ spin transitions shift [Fig. 2(a)], corresponding to a $D$-term shift of the spin Hamiltonian (Eq. 1). Although we observe dc spin resonance shifts of up to $0.8 \mathrm{MHz}$, the resolution of dc-strain detection [29] is constrained by relatively broad $1 / T_{2}{ }^{*}$ spin-resonance linewidths, where $T_{2}{ }^{*}$ is the inhomogeneous spin-dephasing time (1.5 $\mu$ s for these defects [5]). 


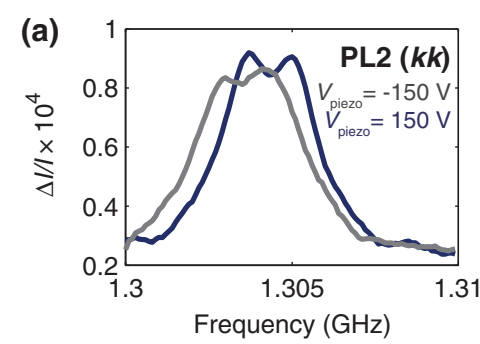

(b)

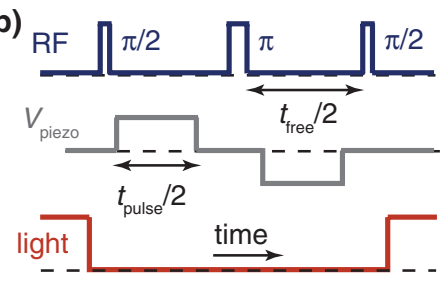

FIG. 2 (color). (a) Strain sensing using dc ODMR of divacancy spins in $\mathrm{SiC}\left(T_{2}{ }^{*}\right.$ limited $)$ at $T=20 \mathrm{~K}$. A strain-induced $0.8 \mathrm{MHz}$ shift to the PL2 spin transition energies is observed, with the split peak within each curve due to a stray $B$ field of 0.35 G. (b) The Hahn-echo pulse scheme used for $T_{2}$-limited ac strain sensing. During the $t_{\text {free }}$ evolution periods, $\pm V_{\text {piezo }}$ pulses phase shift the spin superposition. The final pulse of the Hahnecho sequence projects this phase onto a measurable spin population difference. (c) ac strain sensing data for the neutral divacancies, with $t_{\text {free }}=100 \mu \mathrm{s}, t_{\text {pulse }}=80 \mu \mathrm{s}, T=20 \mathrm{~K}$, and $B=0$. The fits are to single exponentially decaying sine curves and have frequencies of $0.07 \mathrm{~V}^{-1}, 0.07 \mathrm{~V}^{-1}, 0.12 \mathrm{~V}^{-1}$, and $0.16 \mathrm{~V}^{-1}$ for PL1-PL4, respectively. The three curves are offset from the origin for clarity.

We developed an ensemble ac sensing technique that leverages the much longer homogeneous coherence time $\left(T_{2}\right)$ of $\mathrm{SiC}$ divacancies (up to $360 \mu \mathrm{s}$ [7]) to enable more sensitive strain measurements. Here, a Hahn-echo pulse sequence is applied between $m_{s}=0$ and one of the $m_{s}= \pm 1$ spin states $[7,24,33]$. During the free precession segment (of total length $t_{\text {free}}$ ) of this sequence, a synchronized strain perturbation (generated by $\pm V_{\text {piezo }}$ applied to the piezo) causes a strain-induced phase to accumulate between the $m_{s}=0$ and $m_{s}= \pm 1$ spin superposition (Fig. 2(b) and [30]). The final pulse of the Hahn-echo sequence projects this strain-induced phase shift into a spin population difference and, thus, a measurable $\Delta I$. We normalize $\Delta I$ by $\Delta I_{\text {Hahn }}$, the Hahn-echo signal strength with no strain applied. As strain is increased, $\Delta I$ oscillates [Fig. 2(c)] according to this phase shift, demonstrating coherent spinbased strain sensing.

Despite the large uncertainty in our strain calibration, we infer that the spin transitions of PL1-PL4 exhibit strain shifts approximately ranging from $2-4 \mathrm{GHz} /$ strain, and that our ensemble measurements demonstrate sub- $10^{-6}$ strain sensitivity after averaging for two minutes per point. In an ideal measurement, with high optical collection and small background PL, ac strain sensing with neutral divacancies has a projected sensitivity in the $10^{-7} / \sqrt{\mathrm{Hz} \cdot N}$ range (see the Supplemental Material [30]). Optical interferometry is a more precise technique for sensing strain in bulk samples, but it relies on measuring mechanical displacement (with sensitivities down to the $10-\mathrm{pm}$ level [34]). Spin-based strain sensing should, thus, be a competitive technique for measuring strain in micro- and nanostructures, where absolute displacements are extremely small, as well as in applications involving full tensor-strain measurements [32].

To measure the $\mathrm{SiC}$ spin states' response to electric fields, we use the same pulse sequence as for strain measurements (Fig. 2(b) and Ref. [24]), substituting a $c$ axis-oriented voltage applied across the $\mathrm{SiC}$ membrane $\left(V_{\text {mem }}\right)$ for piezoinduced strain [Fig. 3(a)]. As in Fig. 2, $\Delta I$ oscillations are observed, in this case with increasing $V_{\text {mem }}$, which correspond to electric field-induced phase shifts of the spin superposition [Figs. 3(b) and 3(c)]. The basal-oriented defect spins primarily couple to the $c$ axis electric field via $d_{\perp}$ (i.e., $E$ term shifts in Eq. 1), resulting in mirror-image $\Delta I$ oscillations from the two $\Delta m_{s}= \pm 1$ spin-transition frequencies [Fig. 3(b), left] as they are split by the electric field. The $c$ axis-oriented defects have slower $\Delta I$ oscillations [Fig. 3(c)], indicating that their $d_{\|}$parameter (Table I) is significantly smaller than $d_{\perp}$ for the basal-oriented defects. Using PL5, which exhibits strong room-temperature ODMR, we show that $\mathrm{SiC}$ defect spins can sense electric fields at room temperature [Fig. 3(b), right].
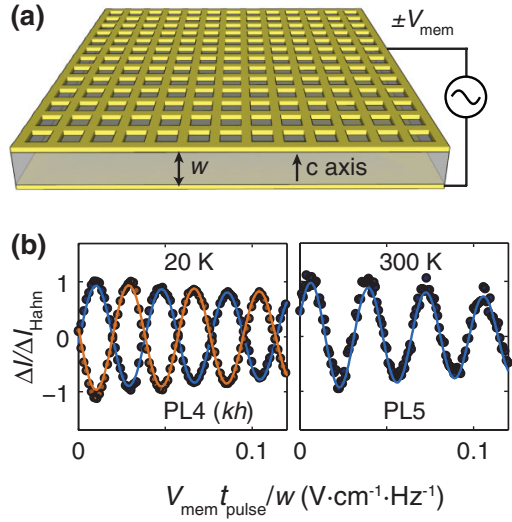

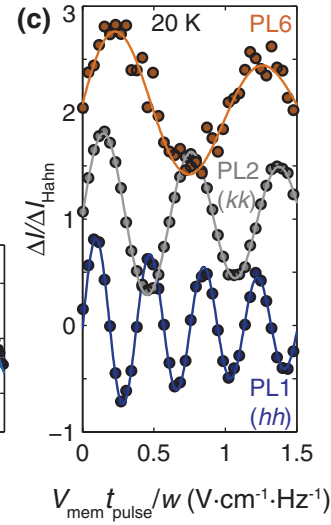

FIG. 3 (color). (a) Electric-field sensing with divacancy and related spins in SiC. Using the same pulse sequence as in Fig. 2(b), but substituting $V_{\text {mem }}$ for $V_{\text {piezo }}, \pm V_{\text {mem }}$ pulses are applied across a $w=53 \mu \mathrm{m}$ thick $\mathrm{SiC}$ membrane, using patterned electrodes that transmit light. As $V_{\text {mem }}$ is increased, $t_{\text {pulse }}$ is held constant at $80 \mu \mathrm{s}$ (for the data at $20 \mathrm{~K}$ ) or $20 \mu \mathrm{s}$ (for the room-temperature data), and $B=0$. (b) ac electric field-sensing measurements for basaloriented defects show mirror image $\Delta I$ oscillations when the higher (blue) and lower (orange) frequency spin-resonance transitions are used (left panel). The PL5 data (right panel) are taken at room temperature. (c) Electric field sensing for the $c$ axis oriented defects at $20 \mathrm{~K}$, with the three curves vertically offset for clarity. The fits are to exponentially decaying sine curves, whose fitted frequencies are the spin-electric field coupling parameters (Table I). 
TABLE I. Experimentally measured and calculated Stark parameters for the PL1-PL6 ground-state-spin Hamiltonians in $4 \mathrm{H}$-SiC, in units of $h \mathrm{~Hz} \mathrm{~cm} / \mathrm{V}$ and a comparison to those for the NV center in diamond, with Ref. [25] used as an experimental reference for the diamond NV center. The experimental (calculated) values of $d_{\|}$are compared to the experimental (calculated) value for the diamond NV center. The rightmost column is $d_{\|}$calculated with only distorted ions due to the piezoelectric effect, i.e., without any extra charge polarization. The experimental uncertainty for the $\mathrm{SiC}$ data is $5 \%$.

\begin{tabular}{lcccc}
\hline \hline & \multicolumn{2}{c}{ Experiment } & \multicolumn{2}{c}{ Theory } \\
Defect/Configuration & $d_{\perp}$ & $d_{\|}$ & $d_{\|}$ & $d_{\|}{ }^{\mathrm{a}}$ \\
\hline NV center & 17 & 0.35 & 0.76 & \\
PL1 $(h h)$ & & 2.65 & 5.2 & 0.38 \\
PL2 $(k k)$ & & 1.61 & 4.2 & 0.23 \\
Ratio of PL1:NV & & $7.6: 1$ & $6.8: 1$ & $0.5: 1$ \\
Ratio of PL2:NV & & $4.6: 1$ & $5.5: 1$ & $0.3: 1$ \\
PL3 $(h k)$ & 32.3 & $<3$ & 0.41 & \\
PL4 $(k h)$ & 28.5 & 0.44 & 0.79 & \\
PL5 & 32.5 & $<3$ & & \\
PL6 & & 0.96 & & \\
\hline \hline
\end{tabular}

${ }^{\mathrm{a} C}$ Calculated with only atom-distortion effect

Room-temperature sensitivity is a significant property for future applications such as biosensing.

An important parameter for high-fidelity sensing is the ODMR contrast $\left(C_{\text {defect }}\right)$ between spin states, defined as the fractional change in PL due to an optically polarized spin population being flipped by $\Delta m_{s}= \pm 1$. We use spectrally resolved ODMR measurements to measure $C_{\text {defect }}$ from ZPLs [Fig. 4(a)], avoiding interference from background PL. Instead of applying $\pi$ pulses to flip spin states, we mix the spin populations with strong continuously applied microwave radiation and weak laser illumination.
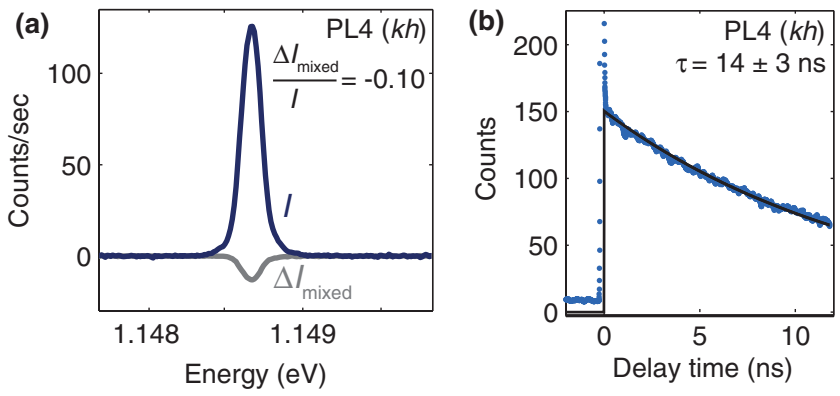

FIG. 4 (color). (a) A plot of the ZPL intensity of PL4 without microwave radiation (blue curve) and the change in PL when strong microwave irradiation is applied to the sample (grey curve). $\Delta I_{\text {mixed }} / I$ is measured to be $-0.10,+0.11,-0.14,-0.10$, -0.24 , and -0.22 for PL1-PL6, respectively. (b) Time-resolved PL from PL4 as a function of delay from an excitation laser pulse (blue dots). The decay constant of the exponential fit (black) is the optical lifetime $(\tau) . T=20 \mathrm{~K}, B=0$. See [30] for the calculation of $C_{\text {defect }}$ from $\Delta I_{\text {mixed }} / I$ and for the other defect species' lifetimes and ODMR contrasts.
Calculating $C_{\text {defect }}$ from the measured fractional change in PL intensity $\left(\Delta I_{\text {mixed }} / I\right)$ requires detailed knowledge of spin mixing dynamics, but we use $3 / 2 \times \Delta I_{\text {mixed }} / I$ as a conservative lower bound for $C_{\text {defect }}$ [30], which is found to range from $0.15-0.21$ for the neutral divacancies (PL1-PL4), and from 0.33-0.36 for PL5 and PL6 at $20 \mathrm{~K}$. These high $C_{\text {defect }}$ values are comparable to that for the NV center in diamond, whose optimized ODMR contrast is typically around $0.3[35,36]$. The optical lifetimes of the $\mathrm{SiC}$ divacancies, another important quantity for spin readout, is found to range from 12-15 ns [Fig. 4(b)], also comparable to that for NV centers in diamond [35]. These favorable ODMR characteristics make SiC defects compelling systems for precision sensing.

In order to understand the interplay between strain-and electric field-induced spin shifts in SiC, we carry out $a b$ initio density-functional calculations of the zero-field splitting parameters in the ground-state Hamiltonian, along with electric- and strain-field perturbations to them. Since our simulations calculate spin-spin interactions between electrons localized to the defects but neglect spin-orbit coupling, we focus on the $c$ axis-oriented divacancies, whose $C_{3 v}$ symmetry implies low spin-orbit coupling. Our simulation methodology [30] provides excellent agreement with the ground state $D^{0}$ values for the $c$ axis-oriented divacancies in $4 \mathrm{H}-\mathrm{SiC}$ as well as for the $\mathrm{NV}$ center in diamond (within 1.5\%). As a corroboration for our simulation methods, we also verify the change in zero-field splitting due to external pressure acting on $\mathrm{NV}$ center in diamond (10.3 MHz/Gpa (cryogenic) calculated vs 14.6 MHz/Gpa (room-temperature) experiment [29]).

The experimental observation that tensile and compressive strain perpendicular to the $c$ axis primarily shifts the $D$ term of the Hamiltonian for the $c$ axis oriented defects [see Fig. 2(a)] is supported by simulations. These show that this strain causes only a small deviation from $C_{3 v}$ symmetry for the defect's electronic orbitals, with their spin exhibiting a correspondingly small $E_{x, y}$ term but a larger $D$ shift of $7 \mathrm{GHz} /$ strain, neglecting Poisson effects. These magnitudes are comparable to the experimentally determined $(2-4 \mathrm{GHz} /$ strain) values, but both are only order-ofmagnitude estimates. For $c$ axis-oriented strain, a $D$ shift of $5 \mathrm{GHz} /$ strain is calculated.

The measured electric field-spin coupling coefficients are consistently found to be higher than those [24,25] for the diamond $\mathrm{NV}$ center, up to 1.9x higher for $d_{\perp}$ and 7.6x higher for $d_{\|}$(Table I). While our simulations overestimate $d_{\|}$in both $\mathrm{SiC}$ and diamond, they accurately corroborate (within 30\%) the experimentally observed relative enhancement of $d_{\|}$for neutral divacancies in $\mathrm{SiC}$ over that for diamond $\mathrm{NV}$ centers.

Two physical effects contribute to $d_{\|}$. First, electric fields distort the positions of atoms in the $\mathrm{SiC}$ lattice neighboring the divacancy. Second, electric fields shift the electron distribution surrounding the defect. Although these effects 
occur simultaneously and cannot be distinguished by experiments alone, simulations can separate the two effects. By calculating $d_{\|}$with a distorted lattice but no extra fieldinduced shifts to electron wave functions, we find $d_{\|}$to be an order of magnitude smaller than its value when electric fields are fully turned on (Table I). Thus, direct field-induced shifts of electron wave functions are primarily responsible for the Stark-shift parameters, not the piezoelectric effect.

The enhanced Stark effect of divacancy spins in SiC over $\mathrm{NV}$-center spins in diamond can be generally understood by the relative electron polarizability in the two materials. The polar crystal bonds in $\mathrm{SiC}$ have high electron polarizability, which can be seen by the material's high (10.0) dielectric constant. Since defect wave functions derive from dangling bond orbitals, they inherit this high polarizability. In turn, this polarizability causes a strong spin response to external electric fields.

Our results show that the spins of neutral divacancies in $\mathrm{SiC}$ can sensitively detect both strain and electric fields. They exhibit high optical polarization [7], high intrinsic ODMR contrasts, and a stronger response to electric fields than those of $\mathrm{NV}$ centers in diamond. In the future, $\mathrm{SiC}$ defects could also be used for temperature sensing [22,23,37-39], particularly since basal-oriented defects exhibit first-order insensitivity to magnetic fields. Moreover, the combination of the spin-strain interactions measured here and the outstanding electromechanical properties of $\mathrm{SiC}$ [27] makes $\mathrm{SiC}$ an excellent material for coupling spins to mechanical resonators [40]. These coupled systems could lead to mechanically induced spin squeezing [41], strong coupling between spins and phonons, and phonon lasing [42].

The authors thank Martin Stevens, Sae Woo Nam, and Richard Mirin at NIST in Boulder, Colorado for providing assistance measuring the optical lifetimes. This work is supported by the AFOSR, DARPA, and NSF. V. I., I. A. A., and A. G. acknowledge the support from Knut and Alice Wallenberg Foundation ("Isotopic Control for Ultimate Material Properties" project) and the National Supercomputer Center (SNIC 001-12-275). A. G. thanks the support from EU Commission (FP7 Grant No. 270197DIAMANT) and the MTA Lenduilet program from the Hungarian Academy of Sciences.

* Corresponding author awsch@uchicago.edu

[1] P. G. Baranov, I. V. Il'in, E. N. Mokhov, M. V. Muzaforova, S. B. Orlinskii, and J. Schmidt, JETP Lett. 82, 441 (2005).

[2] N. T. Son, P. Carlsson, J. ul Hassan, E. Janzén, T. Umeda, J. Isoya, A. Gali, M. Bockstedte, N. Morishita, T. Ohshima, and H. Itoh, Phys. Rev. Lett. 96, 055501 (2006).

[3] J. v. Tol, G. W. Morley, S. Takahashi, D. R. McCamey, C. Boehme, and M. E. Zvanut, Appl. Magn. Reson. 36, 259 (2009).
[4] J. R. Weber, W. F. Koehl, J. B. Varley, A. Janotti, B. B. Buckley, C. G. V. d. Walle, and D. D. Awschalom, Proc. Natl. Acad. Sci. U.S.A. 107, 8513 (2010).

[5] W. F. Koehl, B. B. Buckley, F. J. Heremans, G. Calusine, and D. D. Awschalom, Nature (London) 479, 84 (2011).

[6] D. Riedel, F. Fuchs, H. Kraus, S. Väth, A. Sperlich, V. Dyakonov, A. A. Soltamova, P. G. Baranov, V. A. Ilyin, and G. V. Astakhov, Phys. Rev. Lett. 109, 226402 (2012).

[7] A. L. Falk, B. B. Buckley, G. Calusine, W. F. Koehl, V. V. Dobrovitski, A. Politi, C. A. Zorman, P. X.-L. Feng, and D. D. Awschalom, Nat. Commun. 4, 1819 (2013).

[8] P. G. Baranov, A. P. Bundakova, A. A. Soltamov, S. B. Orlinskii, I. V. Borovykh, R. Zondervan, R. Verberk, and J. Schmidt, Phys. Rev. B 83, 125203 (2011).

[9] V. A. Soltamov, A. A. Soltamova, P. G. Baranov, and I. I. Proskuryakov, Phys. Rev. Lett. 108, 226402 (2012).

[10] V. V. Dobrovitski, G. D. Fuchs, A. L. Falk, C. Santori, and D. D. Awschalom, Annu. Rev. Condens. Matter Phys. 4, 23 (2013).

[11] W. E. Carlos, E. R. Glaser, and B. V. Shanabrook, Physica (Amsterdam) 340B-342B, 151 (2003).

[12] B. Magnusson and E. Janzen, Mater. Sci. Forum 483-485, 341 (2005).

[13] W. E. Carlos, N. Y. Garces, E. R. Glaser, and M. A. Fanton, Phys. Rev. B 74, 235201 (2006).

[14] A. Gali, Phys. Status Solidi B 248, 1337 (2011).

[15] H. Bernien, B. Hensen, W. Pfaff, G. Koolstra, M. S. Blok, L. Robledo, T. H. Taminiau, M. Markham, D. J. Twitchen, L. Childress, and R. Hanson, Nature (London) 497, 86 (2013).

[16] H. J. Mamin, M. Kim, M. H. Sherwood, C. T. Rettner, K. Ohno, D. D. Awschalom, and D. Rugar, Science 339, 557 (2013).

[17] T. Staudacher, F. Shi, S. Pezzagna, J. Meijer, J. Du, C. A. Meriles, F. Reinhard, and J. Wrachtrup, Science 339, 561 (2013).

[18] S. E. Saddow, and A. K. Agarwal, Advances in Silicon Carbide Processing and Applications (Artech House, Norwood, MA, 2004).

[19] L. Liu and J. H. Edgar, Mater. Sci. Eng. R 37, 61 (2002).

[20] C. Berger, Z. Song, X. Li, W. Wu, N. Brown, C. Naud, D. Mayou, T. Li, J. Hass, A. N. Marchenkov, E. H. Congrad, P. N. First, and W. A. d. Heer, Science 312, 1191 (2006).

[21] S. Castelletto, B. C. Johnson, V. Ivady, N. Stavrias, T. Umeda, A. Gali, and T. Oshima, Nat. Mater. 13, 151 (2014).

[22] G. Kucsko, P. C. Maurer, N. Y. Yao, M. Kubo, N. J. Noh, P. K. Lo, H. Park, and M. D. Lukin, Nature (London) 500, 54 (2013).

[23] D. M. Toyli, C. F.d.1. Casas, D. J. Christle, V. V. Dobrovitski, and D. D. Awschalom, Proc. Natl. Acad. Sci. U.S.A. 110, 8417 (2013).

[24] F. Dolde, H. Fedder, M. W. Doherty, T. Nobauer, F. Rempp, G. Balasubramanian, T. Wolf, F. Reinhard, L. C. L. Hollenberg, F. Jelezko, and J. Wrachtrup, Nat. Phys. 7, 459 (2011).

[25] E. v. Oort and M. Glasbeek, Chem. Phys. Lett. 168, 529 (1990).

[26] S. Saddow, Silicon Carbide Biotechnology: A Biocompatible Semiconductor for Advanced Biomedical Devices and Applications (Elsevier Science, Waltham, 2011).

[27] Y. T. Yang, Appl. Phys. Lett. 78, 162 (2001). 
[28] V. S. Vainer and V. A. Ilyin, Sov. Phys. Solid State 23, 2126 (1981).

[29] M. W. Doherty, V. V. Struzhkin, D. A. Simpson, L. P. McGuinness, Y. Meng, A. Stacey, T. J. Karle, R. J. Hemley, N. B. Manson, L. C. L. Hollenberg, and S. Prawer, Phys. Rev. Lett. 112, 047601 (2014).

[30] See Supplemental Material at http://link.aps.org/supplemental/ 10.1103/PhysRevLett.112.187601 for additional data, experimental details, and theoretical methodology.

[31] G. Davies and F. Hamer, Proc. R. Soc. A 348, 285 (1976).

[32] F. Grazioso, B. R. Patton, P. Delaney, M. L. Markham, D. J. Twitchen, and J. M. Smith, Appl. Phys. Lett. 103, 101905 (2013).

[33] J. M. Taylor, P. Cappellaro, L. Childress, L. Jiang, D. Budker, P. R. Hemmer, A. Yacoby, W. Walsworth, and M. D. Lukin, Nat. Phys. 4, 810 (2008).

[34] J. L. a. E. Kessler, Rev. Sci. Instrum. 71, 2669 (2000).

[35] L. Robledo, H. Bernien, T. v. d. Sar, and R. Hanson, New J. Phys. 13, 025013 (2011).
[36] M. Steiner, P. Neumann, J. Beck, F. Jelezko, and J. Wrachtrup, Phys. Rev. B 81, 035205 (2010).

[37] V. M. Acosta, E. Bauch, M. P. Ledbetter, A. Waxman, L.-S. Bouchard, and D. Budker, Phys. Rev. Lett. 104, 070801 (2010).

[38] P. Neumann, I. Jakobi, F. Dolde, C. Burk, R. Reuter, G. Waldherr, J. Honert, T. Wolf, A. Brunner, J. H. Shim, D. Suter, H. Sumiya, J. Isoya, and J. Wrachtrup, Nano Lett. 13, 2738 (2013).

[39] D. M. Toyli, D. J. Christle, A. Alkauskas, B. B. Buckley, C. G. Van de Walle, and D. D. Awschalom, Phys. Rev. X 2, 031001 (2012).

[40] E. R. MacQuarrie, T. A. Gosavi, N. R. Jungwirth, S. A. Bhave, and G. D. Fuchs, Phys. Rev. Lett. 111, 227602 (2013).

[41] S. D. Bennett, N. Y. Yao, J. Otterbach, P. Rabl, and M. D. Lukin, Phys. Rev. Lett. 110, 156402 (2013).

[42] K. V. Kepesidis, S. D. Bennett, S. Portolan, M. D. Lukin, and P. Rabl, Phys. Rev. B 88, 064105 (2013). 